\title{
A van der Waals Correction to the Physisorption of Graphene on Metal Surfaces
}

\author{
Supporting Information
}

Hong Tang, Jianmin Tao, and Adrienn Ruzsinszky, John P. Perdew

Department of Physics, Temple University, Philadelphia, Pennsylvania 19122, USA 

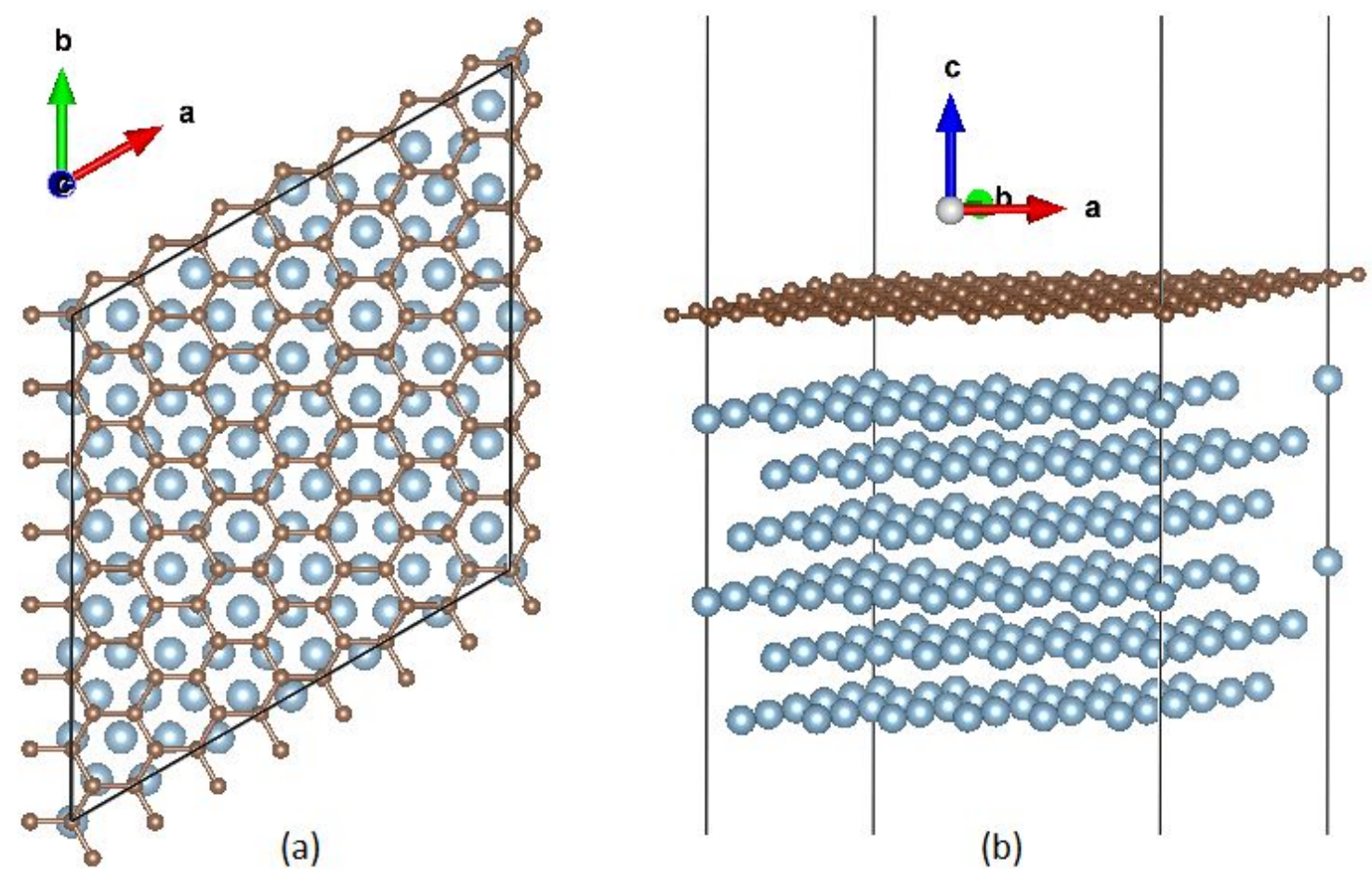

(b)

Figure S1. Optimized atomic structure of graphene on the $(1,1,1)$ surface of Al with the DFT PBE calculation. (a) top view and (b) side view. The brown and blue balls stand for $\mathrm{C}$ and $\mathrm{Al}$ atoms, respectively. 


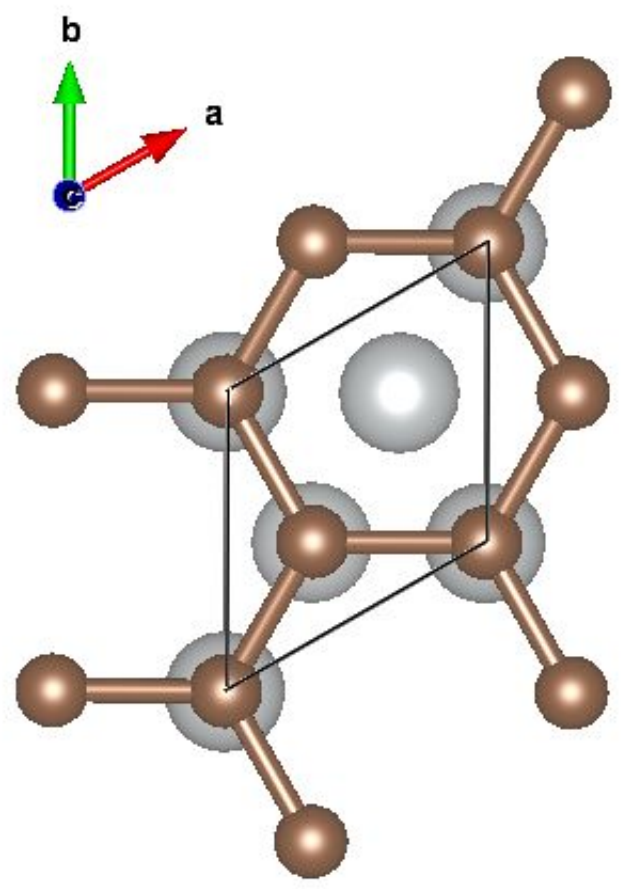

(a)

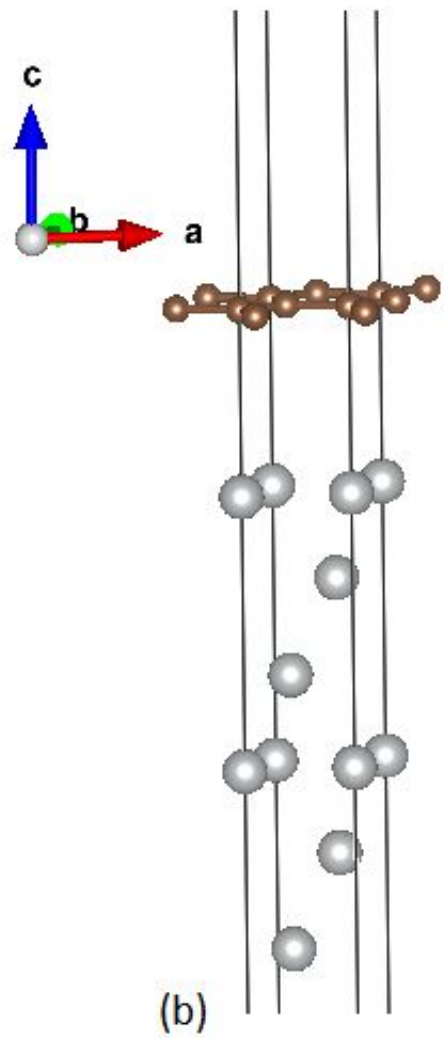

(b)

Figure S2. Optimized atomic structure of graphene on the $(1,1,1)$ surface of Ni with the DFT PBE calculation. (a) top view and (b) side view. The brown and gray balls stand for $\mathrm{C}$ and $\mathrm{Ni}$ atoms, respectively. 


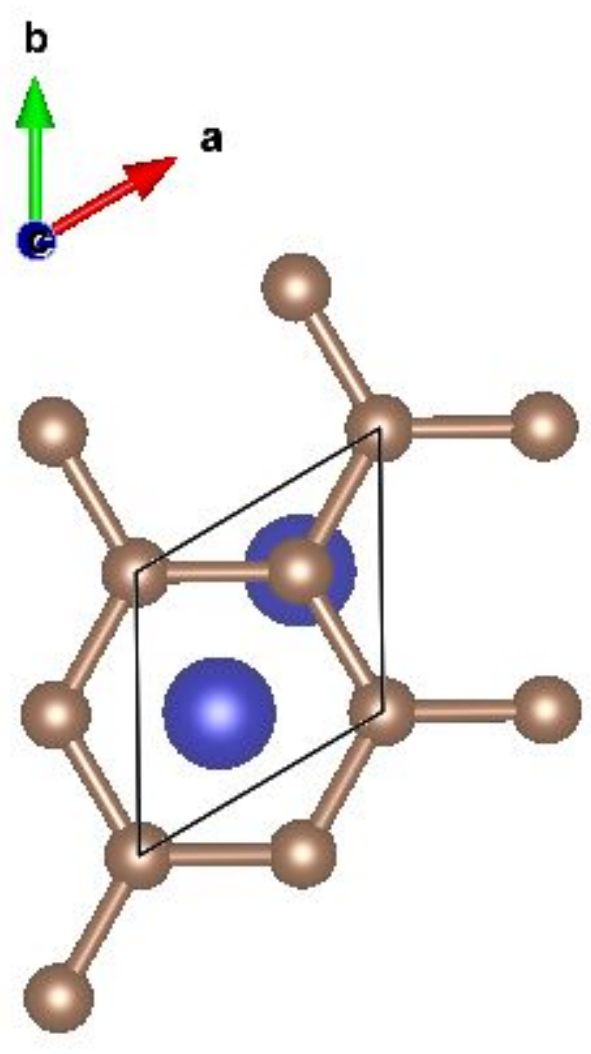

(a)

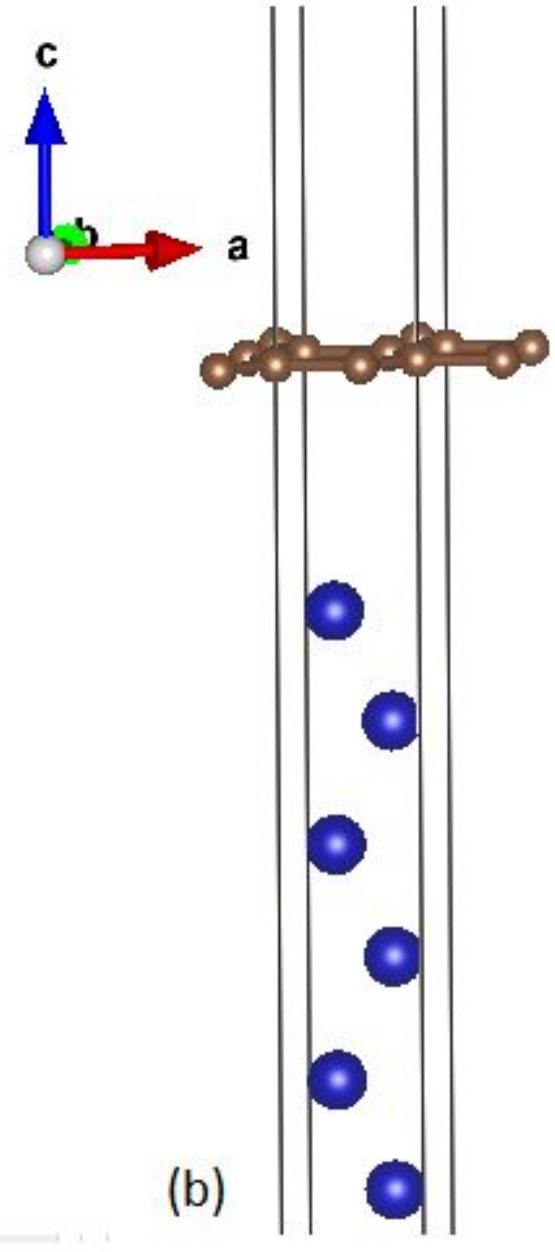

(b)

Figure S3. Optimized atomic structure of graphene on the $(0,0,0,1)$ surface of Co with the DFT PBE calculation. (a) top view and (b) side view. The brown and blue balls stand for $\mathrm{C}$ and $\mathrm{Co}$ atoms, respectively. 

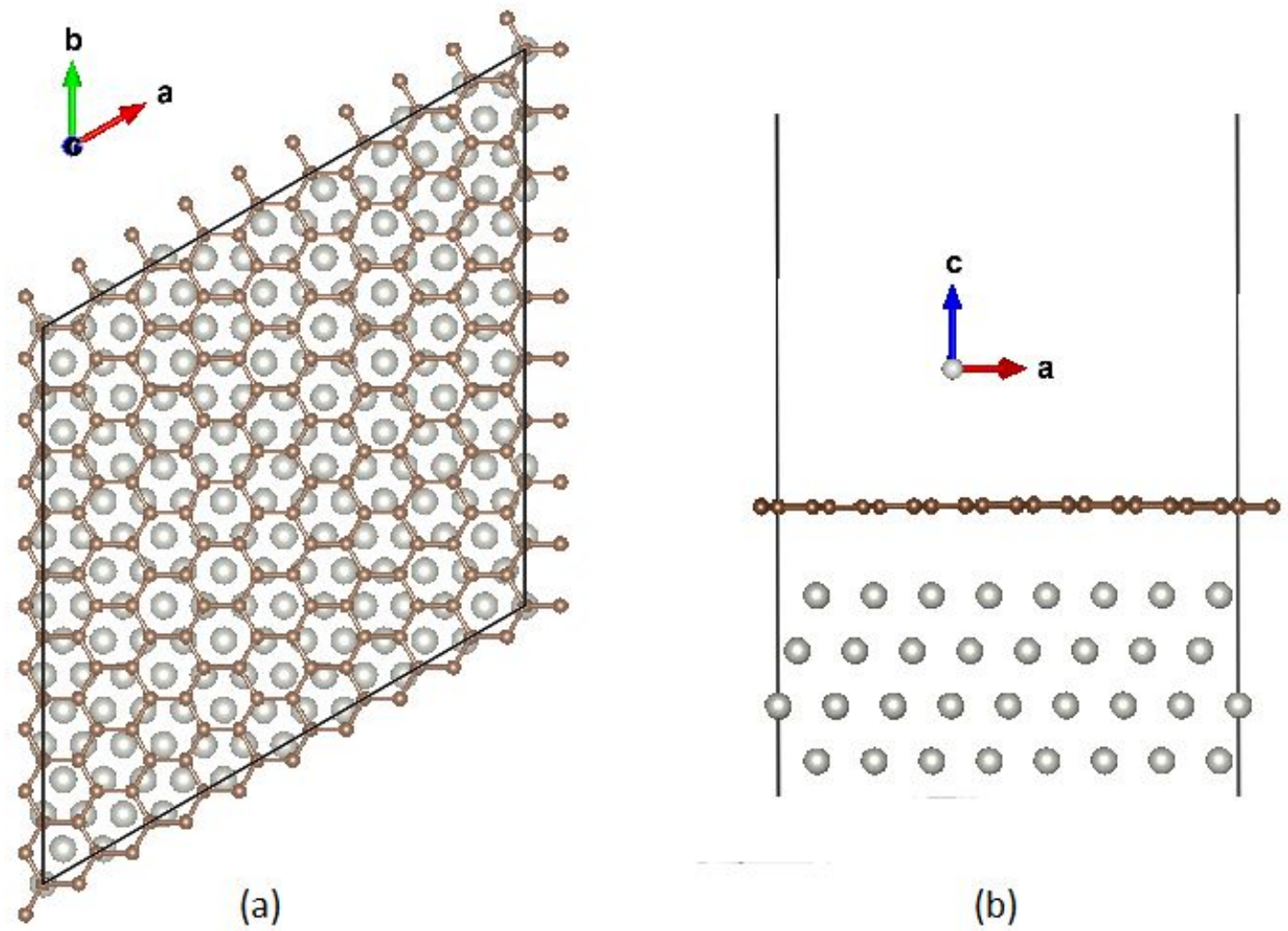

(b)

Figure S4. Optimized atomic structure of graphene on the $(1,1,1)$ surface of Pd with the DFT PBE calculation. (a) top view and (b) side view. The brown and gray balls stand for $\mathrm{C}$ and $\mathrm{Pd}$ atoms, respectively. 

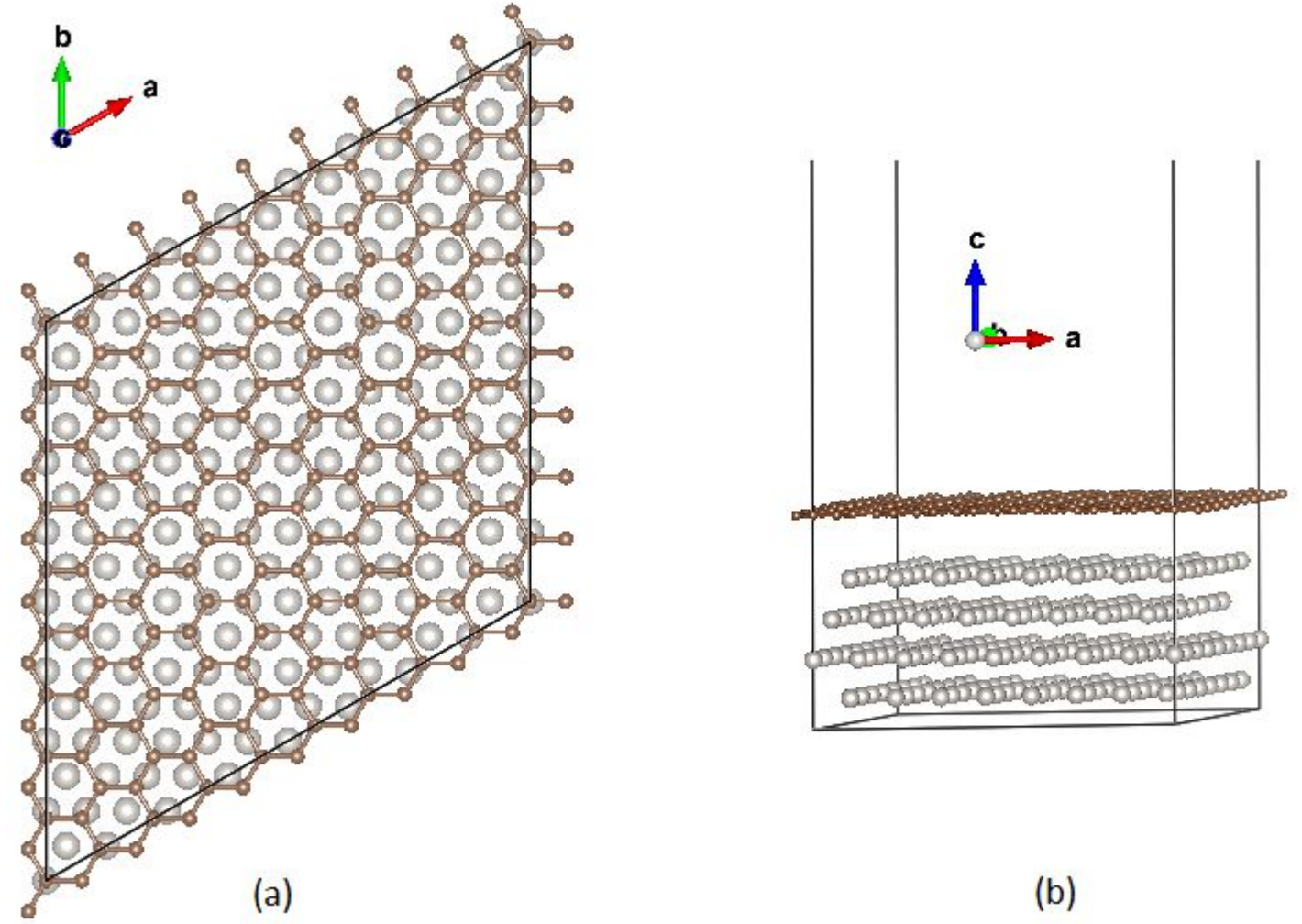

Figure S5. Optimized atomic structure of graphene on the $(1,1,1)$ surface of Pt with the DFT PBE calculation. (a) top view and (b) side view. The brown and gray balls stand for $\mathrm{C}$ and $\mathrm{Pt}$ atoms, respectively. 


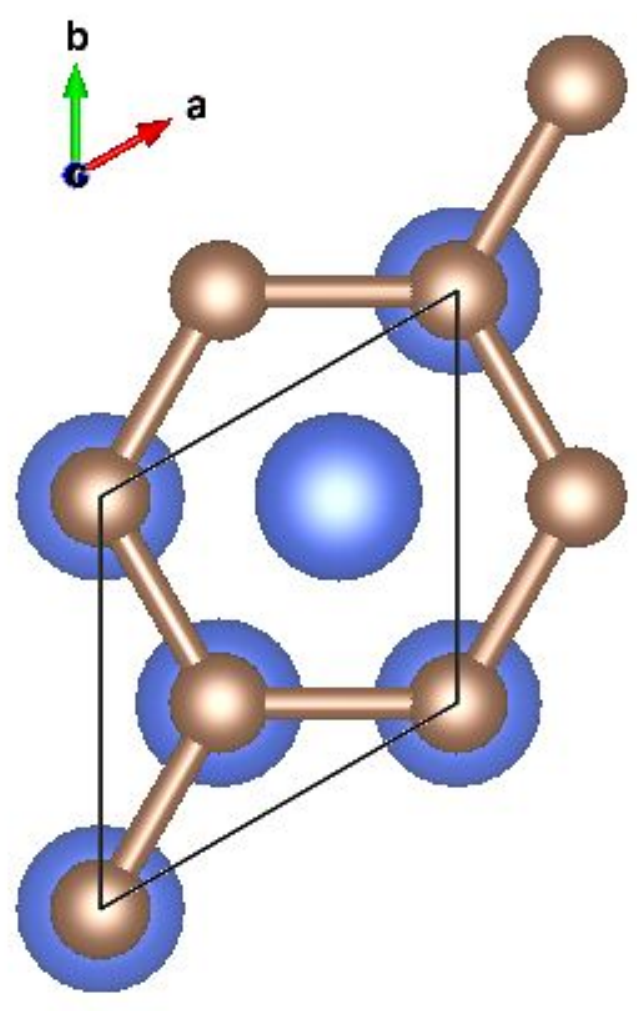

(a)

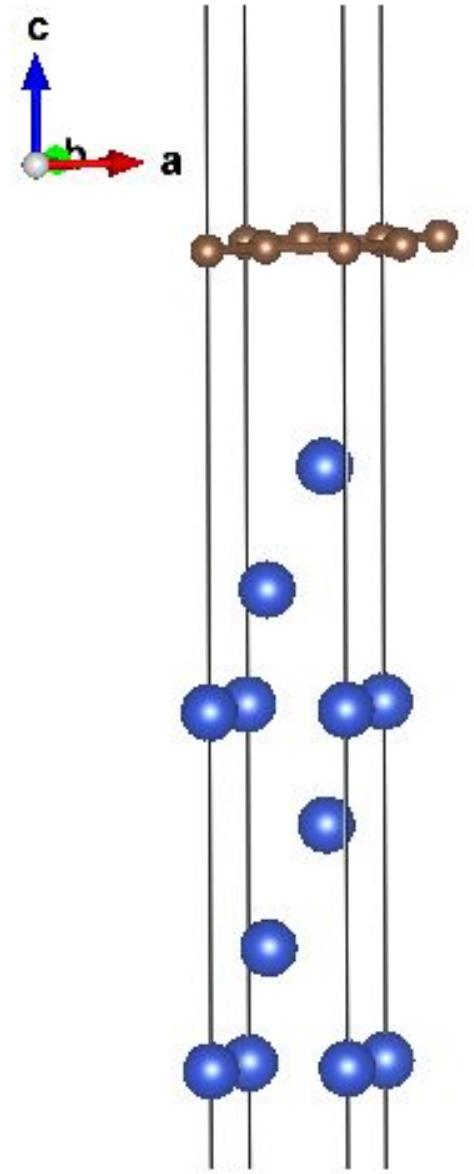

(b)

Figure S6. Optimized atomic structure of graphene on the $(1,1,1)$ surface of $\mathrm{Cu}$ with the DFT PBE calculation. (a) top view and (b) side view. The brown and blue balls stand for $\mathrm{C}$ and $\mathrm{Cu}$ atoms, respectively. 


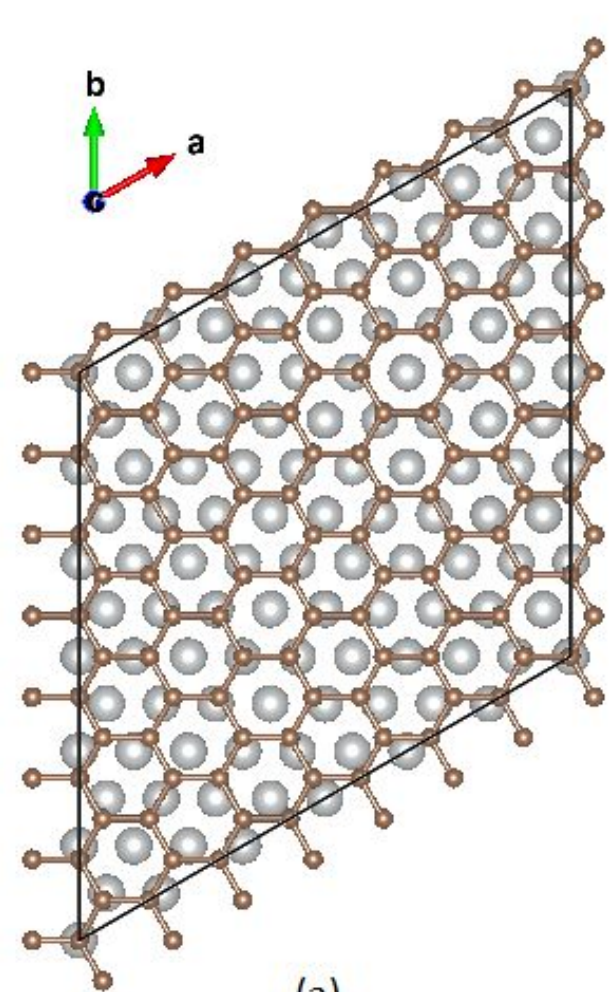

(a)

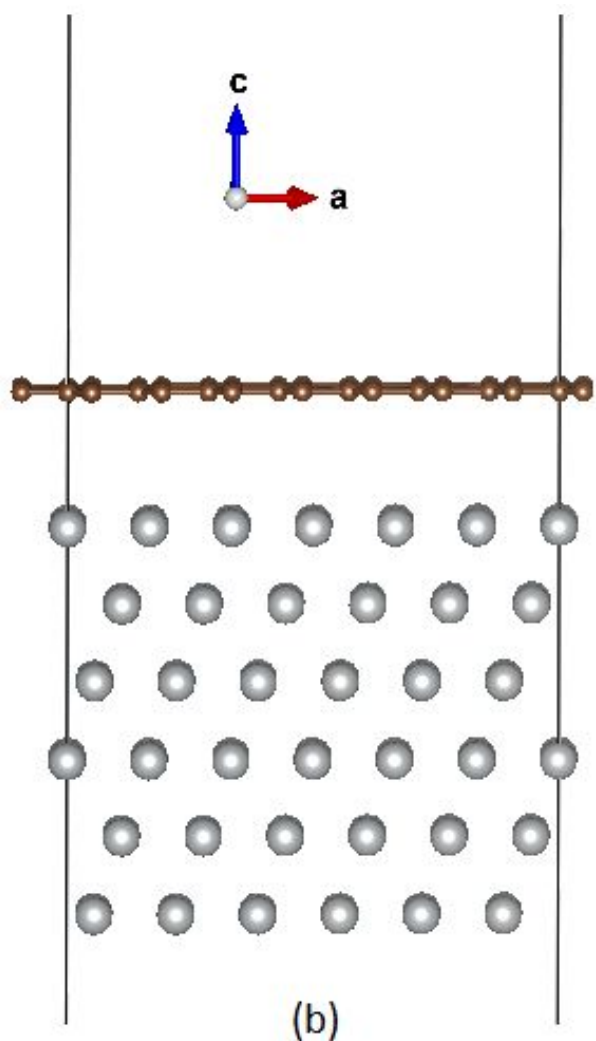

(b)

Figure S7. Optimized atomic structure of graphene on the $(1,1,1)$ surface of Ag with the DFT PBE calculation. (a) top view and (b) side view. The brown and gray balls stand for $\mathrm{C}$ and $\mathrm{Ag}$ atoms, respectively. 


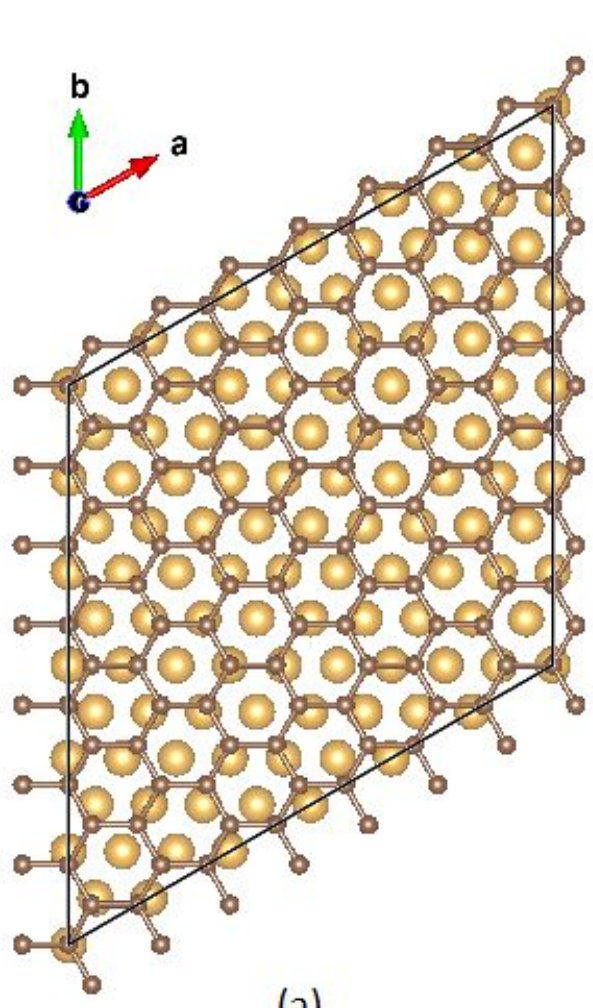

(a)

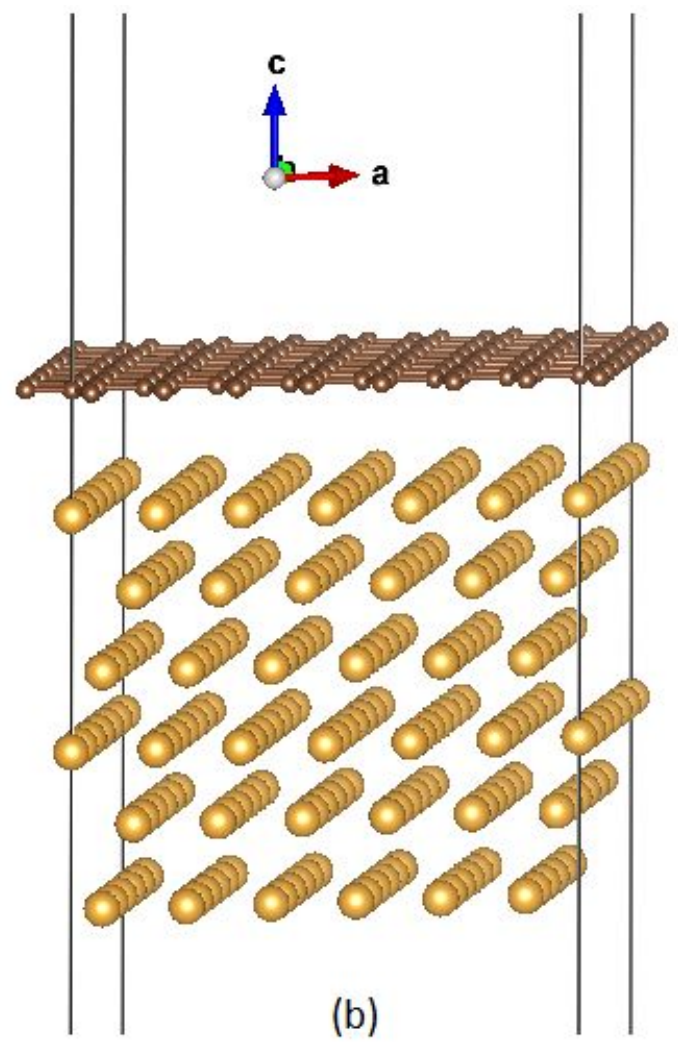

(b)

Figure S8. Optimized atomic structure of graphene on the $(1,1,1)$ surface of Au with the DFT PBE calculation. (a) top view and (b) side view. The brown and gold balls stand for $\mathrm{C}$ and $\mathrm{Au}$ atoms, respectively. 


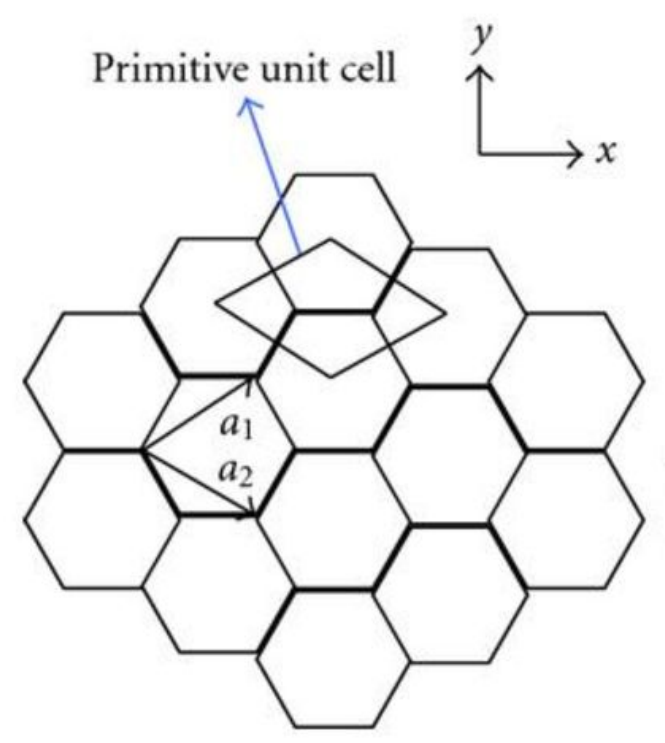

Figure S9. The geometry of the primitive unit cell of the graphene layer.

Table S1. Input parameters (in a.u., i.e., $e=m_{e}=\hbar=1$ ) used to calculate the dielectric functions for metals. For $\mathrm{Cu}, \mathrm{Ag}$ and $\mathrm{Au}$, values for $r_{s}, m_{\text {opt }}, \omega_{0}$, and $\Omega$ are taken from Ref. [1], and $r_{s}^{f}$ is calculated from $r_{s}^{f}=\left(m_{o p t}\right)^{1 / 3} r_{s}$. For Ni, Co, Pd, and Pt, values for $r_{s}, m_{o p t}, \omega_{0}$, and $\Omega$ are extrapolated, as explained in Ref. [2]. The $r_{s}$ of Al is evaluated from the jellium model.

\begin{tabular}{l|cccccc}
\hline \hline Simple metal & $r_{s}$ & $m_{\text {opt }}$ & $\omega_{0}$ & $\Omega$ & $\lambda$ & $d_{\perp}(0)$ \\
\hline $\mathrm{Al}$ & 2.07 & - & - & - & 0.469 & 1.587 \\
\hline & & & & & & \\
\hline Metals & $r_{s}^{f}$ & $m_{\text {opt }}$ & $\omega_{0}$ & $\Omega$ & $\lambda$ & $d_{\perp}^{f}(0)$ \\
\hline $\mathrm{Ni}$ & 3.04 & 1.60 & 0.344 & 0.640 & 0.542 & 1.424 \\
$\mathrm{Co}$ & 3.05 & 1.59 & 0.343 & 0.655 & 0.542 & 1.423 \\
$\mathrm{Pd}$ & 3.07 & 1.22 & 0.350 & 1.050 & 0.543 & 1.420 \\
$\mathrm{Pt}$ & 3.06 & 1.17 & 0.355 & 1.070 & 0.543 & 1.422 \\
$\mathrm{Cu}$ & 3.06 & 1.50 & 0.346 & 0.748 & 0.543 & 1.422 \\
$\mathrm{Ag}$ & 2.98 & 0.96 & 0.544 & 1.001 & 0.538 & 1.433 \\
$\mathrm{Au}$ & 3.00 & 0.99 & 0.386 & 1.051 & 0.539 & 1.430 \\
\hline
\end{tabular}




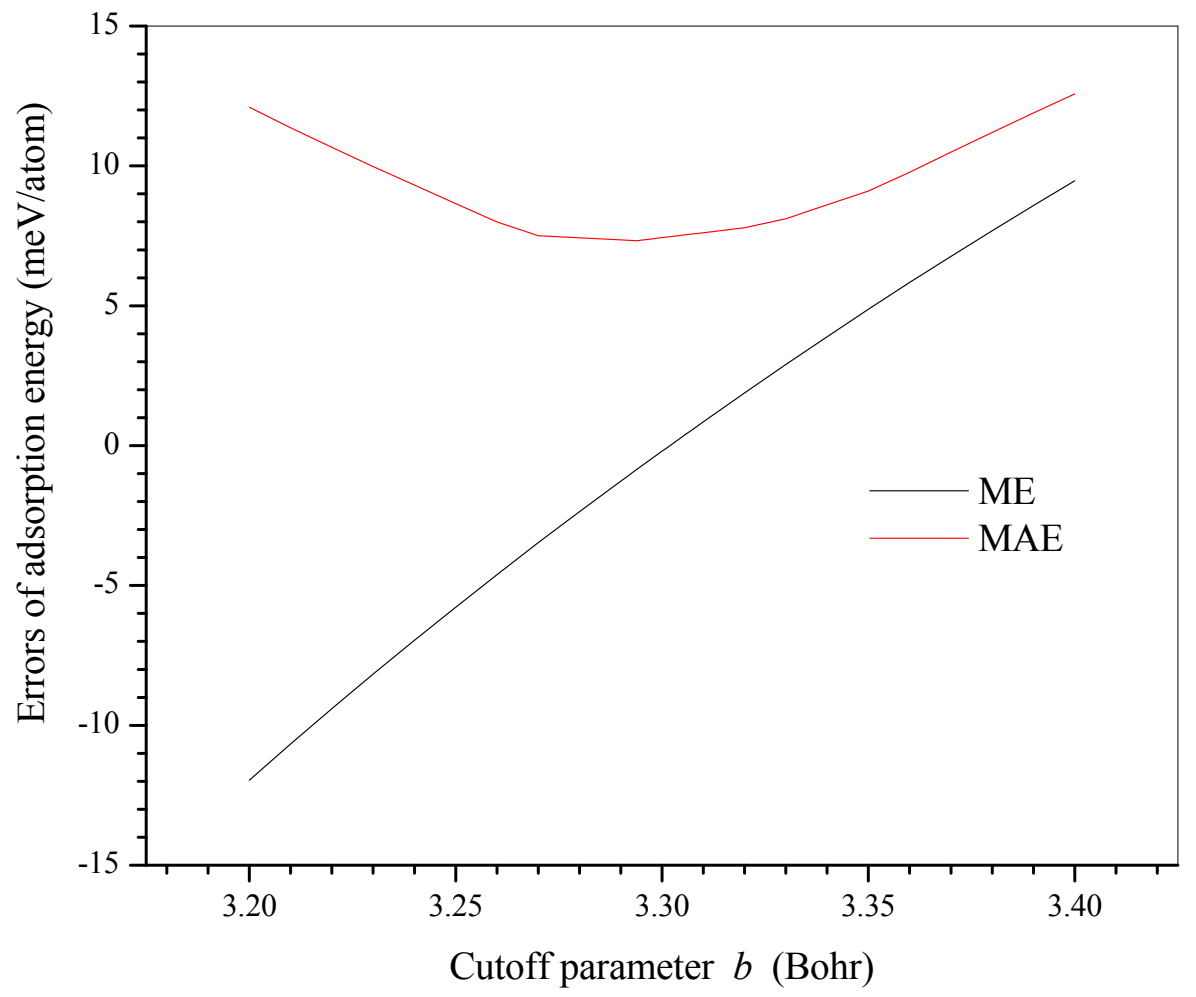

Figure S10. The mean error (ME) and mean absolute error (MAE) of adsorption energy for graphene on $\mathrm{Al}(1,1,1), \mathrm{Ni}(1,1,1), \mathrm{Co}(0,0,0,1), \mathrm{Pd}(1,1,1), \mathrm{Pt}(1,1,1), \mathrm{Cu}(1,1,1), \mathrm{Ag}(1,1,1)$, and $\mathrm{Au}(1,1,1)$ surfaces, compared to the reference values of RPA calculations as a function of cutoff parameter $b$. The optimal cutoff parameter $b$ is 3.30 Bohr. 
Table S2. Adsorption energies from the DFT calculation with the PBE functional, from the vdW correction, and from their addition, denoted as total adsorption $E_{\text {adsorp }}$ for graphene adsorbed on the $\mathrm{Al}(1,1,1)$ surface at different distances between graphene and metal surface, at optimized cutoff parameter $b=3.30$ Bohr. Units: Angstrom for distance and meV/carbon atom for energy.

\begin{tabular}{lrrr}
\hline$d$ & \multicolumn{1}{l}{$E_{P B E}$} & \multicolumn{1}{c}{$E_{v d W}$} & $E_{\text {adsorp }}$ \\
\hline 2.09 & 288.25 & -157.00 & 131.25 \\
2.19 & 236.18 & -150.82 & 85.36 \\
2.29 & 196.41 & -143.11 & 53.31 \\
2.39 & 164.04 & -134.59 & 29.45 \\
2.49 & 137.07 & -125.98 & 11.09 \\
2.59 & 113.08 & -117.78 & -4.70 \\
2.69 & 91.78 & -110.29 & -18.51 \\
2.79 & 73.08 & -103.61 & -30.53 \\
2.89 & 57.03 & -97.72 & -40.69 \\
2.99 & 43.48 & -92.55 & -49.07 \\
3.09 & 31.85 & -88.00 & -56.16 \\
3.19 & 21.91 & -83.97 & -62.07 \\
3.29 & 13.66 & -80.36 & -66.70 \\
3.39 & 7.01 & -77.07 & -70.06 \\
3.49 & 1.79 & -74.02 & -72.23 \\
3.60 & -2.12 & -71.13 & -73.25 \\
3.70 & -4.74 & -68.35 & -73.09 \\
3.80 & -6.51 & -65.61 & -72.12 \\
3.90 & -7.64 & -62.89 & -70.52 \\
4.00 & -8.27 & -60.15 & -68.42 \\
4.10 & -8.62 & -57.38 & -66.00 \\
4.20 & -8.79 & -54.58 & -63.37 \\
4.30 & -8.85 & -51.75 & -60.60 \\
4.40 & -8.83 & -48.91 & -57.74 \\
4.50 & -8.77 & -46.10 & -54.86 \\
\hline & & &
\end{tabular}


Table S3. Adsorption energies from the DFT calculation with the PBE functional, from the vdW correction, and from their addition, denoted as total adsorption $E_{\text {adsorp }}$ for graphene adsorbed on the $\mathrm{Ni}(1,1,1)$ surface at different distances between graphene and metal surface, at optimized cutoff parameter $b=3.30$ Bohr. Units: Angstrom for distance and meV/carbon atom for energy.

\begin{tabular}{lrrr}
\hline$d$ & $E_{P B E}$ & $E_{v d W}$ & $E_{\text {adsorp }}$ \\
\hline 2.95 & 16.61 & -84.03 & -67.42 \\
3.03 & 12.74 & -81.09 & -68.35 \\
3.12 & 9.33 & -78.31 & -68.99 \\
3.20 & 6.31 & -75.65 & -69.34 \\
3.29 & 3.73 & -73.08 & -69.35 \\
3.37 & 1.55 & -70.55 & -69.00 \\
3.45 & -0.24 & -68.04 & -68.28 \\
3.54 & -1.68 & -65.53 & -67.21 \\
3.62 & -2.83 & -63.00 & -65.84 \\
3.71 & -3.71 & -60.46 & -64.17 \\
3.79 & -4.36 & -57.89 & -62.25 \\
3.87 & -4.82 & -55.31 & -60.14 \\
3.96 & -5.15 & -52.73 & -57.88 \\
4.04 & -5.33 & -50.16 & -55.49 \\
4.13 & -5.43 & -47.61 & -53.04 \\
4.21 & -5.46 & -45.11 & -50.57 \\
4.29 & -5.40 & -42.66 & -48.07 \\
4.38 & -5.34 & -40.29 & -45.63 \\
4.46 & -5.22 & -38.01 & -43.22 \\
4.55 & -5.09 & -35.82 & -40.91 \\
4.63 & -4.93 & -33.73 & -38.66 \\
4.71 & -4.77 & -31.74 & -36.52 \\
4.80 & -4.60 & -29.87 & -34.47 \\
4.88 & -4.42 & -28.10 & -32.52 \\
4.97 & -4.22 & -26.44 & -30.66 \\
5.05 & -4.07 & -24.88 & -28.95 \\
5.13 & -3.91 & -23.42 & -27.33 \\
5.22 & -3.73 & -22.06 & -25.78 \\
\hline & & & \\
& & \\
3.93
\end{tabular}


Table S4. Adsorption energies from the DFT calculation with the PBE functional, from the vdW correction, and from their addition, denoted as total adsorption $E_{\text {adsorp }}$ for graphene adsorbed on the Co $(0,0,0,1)$ surface at different distances between graphene and metal surface, at optimized cutoff parameter $b=3.30$ Bohr. Units: Angstrom for distance and meV/carbon atom for energy.

\begin{tabular}{lccr}
\hline$d$ & $E_{P B E}$ & $E_{\text {vdW }}$ & $E_{\text {adsorp }}$ \\
\hline 2.84 & 80.69 & -87.91 & -7.22 \\
2.93 & 64.40 & -84.51 & -20.12 \\
3.02 & 50.32 & -81.36 & -31.04 \\
3.11 & 38.48 & -78.39 & -39.91 \\
3.20 & 28.72 & -75.54 & -46.82 \\
3.29 & 20.90 & -72.78 & -51.87 \\
3.38 & 14.83 & -70.05 & -55.22 \\
3.47 & 10.03 & -67.34 & -57.30 \\
3.56 & 6.57 & -64.61 & -58.04 \\
3.65 & 4.00 & -61.86 & -57.86 \\
3.74 & 2.10 & -59.09 & -56.99 \\
3.83 & 0.69 & -56.30 & -55.61 \\
3.92 & -0.33 & -53.50 & -53.83 \\
4.01 & -1.06 & -50.72 & -51.78 \\
4.10 & -1.59 & -47.96 & -49.55 \\
4.19 & -1.94 & -45.26 & -47.20 \\
4.28 & -2.14 & -42.62 & -44.77 \\
4.37 & -2.29 & -40.08 & -42.37 \\
4.46 & -2.35 & -37.63 & -39.98 \\
4.55 & -2.32 & -35.30 & -37.63 \\
4.64 & -2.29 & -33.09 & -35.38 \\
4.73 & -2.26 & -31.01 & -33.26 \\
4.82 & -2.18 & -29.04 & -31.22 \\
4.91 & -2.08 & -27.21 & -29.29 \\
5.00 & -1.99 & -25.49 & -27.48 \\
5.09 & -1.88 & -23.89 & -25.77 \\
5.18 & -1.77 & -22.40 & -24.17 \\
5.27 & -1.65 & -21.02 & -22.67 \\
5.36 & -1.55 & -19.73 & -21.29 \\
5.45 & -1.42 & -18.54 & -19.96 \\
5.54 & -1.34 & -17.43 & -18.78 \\
\hline & & &
\end{tabular}


Table S5. Adsorption energies from the DFT calculation with the PBE functional, from the vdW correction, and from their addition, denoted as total adsorption $E_{\text {adsorp }}$ for graphene adsorbed on the $\mathrm{Pd}(1,1,1)$ surface at different distances between graphene and metal surface, at optimized cutoff parameter $b=3.30$ Bohr. Units: Angstrom for distance and meV/carbon atom for energy.

\begin{tabular}{lrrr}
\hline$d$ & $E_{P B E}$ & $E_{v d W}$ & $E_{\text {adsorp }}$ \\
\hline 2.40 & 69.86 & -147.62 & -77.76 \\
2.51 & 56.12 & -138.66 & -82.54 \\
2.61 & 45.65 & -130.76 & -85.12 \\
2.71 & 36.89 & -123.82 & -86.93 \\
2.81 & 28.98 & -117.70 & -88.71 \\
2.91 & 21.71 & -112.25 & -90.53 \\
3.02 & 15.21 & -107.33 & -92.12 \\
3.12 & 9.66 & -102.82 & -93.16 \\
3.22 & 5.11 & -98.60 & -93.49 \\
3.32 & 1.53 & -94.58 & -93.04 \\
3.42 & -1.20 & -90.66 & -91.86 \\
3.53 & -3.23 & -86.78 & -90.01 \\
3.63 & -4.68 & -82.90 & -87.57 \\
3.73 & -5.67 & -78.98 & -84.66 \\
3.83 & -6.32 & -75.03 & -81.35 \\
3.93 & -6.70 & -71.04 & -77.75 \\
4.04 & -6.89 & -67.05 & -73.93 \\
4.14 & -6.93 & -63.07 & -70.00 \\
4.24 & -6.87 & -59.16 & -66.03 \\
4.34 & -6.74 & -55.34 & -62.08 \\
4.44 & -6.56 & -51.65 & -58.21 \\
\hline & & &
\end{tabular}


Table S6. Adsorption energies from the DFT calculation with the PBE functional, from the vdW correction, and from their addition, denoted as total adsorption $E_{\text {adsorp }}$ for graphene adsorbed on the $\mathrm{Pt}(1,1,1)$ surface at different distances between graphene and metal surface, at optimized cutoff parameter $b=3.30$ Bohr. Units: Angstrom for distance and meV/carbon atom for energy.

\begin{tabular}{lrrr}
\hline$d$ & \multicolumn{1}{l}{$E_{P B E}$} & \multicolumn{1}{c}{$E_{v d W}$} & $E_{\text {adsorp }}$ \\
\hline 2.40 & 201.31 & -150.74 & 50.57 \\
2.50 & 162.10 & -141.48 & 20.62 \\
2.60 & 128.71 & -133.30 & -4.60 \\
2.70 & 100.07 & -126.12 & -26.05 \\
2.81 & 75.86 & -119.79 & -43.93 \\
2.91 & 55.78 & -114.17 & -58.39 \\
3.01 & 39.44 & -109.12 & -69.68 \\
3.11 & 26.40 & -104.50 & -78.11 \\
3.21 & 16.20 & -100.21 & -84.00 \\
3.32 & 8.42 & -96.12 & -87.70 \\
3.42 & 2.64 & -92.16 & -89.52 \\
3.52 & -1.56 & -88.25 & -89.81 \\
3.62 & -4.54 & -84.35 & -88.89 \\
3.72 & -6.60 & -80.42 & -87.02 \\
3.83 & -7.98 & -76.45 & -84.42 \\
3.93 & -8.84 & -72.44 & -81.28 \\
4.03 & -9.34 & -68.41 & -77.75 \\
4.13 & -9.56 & -64.41 & -73.97 \\
4.23 & -9.57 & -60.45 & -70.02 \\
4.34 & -9.50 & -56.58 & -66.07 \\
4.44 & -9.31 & -52.83 & -62.14 \\
4.54 & -9.06 & -49.24 & -58.30 \\
\hline & & &
\end{tabular}


Table S7. Adsorption energies from the DFT calculation with the PBE functional, from the vdW correction, and from their addition, denoted as total adsorption $E_{\text {adsorp }}$ for graphene adsorbed on the $\mathrm{Cu}(1,1,1)$ surface at different distances between graphene and metal surface, at optimized cutoff parameter $b=3.30$ Bohr. Units: Angstrom for distance and meV/carbon atom for energy.

\begin{tabular}{|c|c|c|c|}
\hline$d$ & $E_{P B E}$ & $E_{v d W}$ & $E_{\text {adsorp }}$ \\
\hline 2.13 & 352.98 & -145.20 & 207.78 \\
\hline 2.23 & 292.19 & -135.75 & 156.44 \\
\hline 2.33 & 239.61 & -127.00 & 112.61 \\
\hline 2.44 & 195.38 & -119.14 & 76.23 \\
\hline 2.54 & 157.70 & -112.19 & 45.52 \\
\hline 2.64 & 125.14 & -106.07 & 19.07 \\
\hline 2.74 & 96.58 & -100.69 & -4.11 \\
\hline 2.84 & 72.56 & -95.91 & -23.35 \\
\hline 2.95 & 54.42 & -91.62 & -37.21 \\
\hline 3.05 & 40.51 & -87.72 & -47.20 \\
\hline 3.15 & 29.52 & -84.09 & -54.56 \\
\hline 3.25 & 20.85 & -80.64 & -59.79 \\
\hline 3.35 & 14.21 & -77.31 & -63.11 \\
\hline 3.46 & 9.15 & -74.04 & -64.89 \\
\hline 3.56 & 5.76 & -70.77 & -65.02 \\
\hline 3.66 & 3.24 & -67.49 & -64.24 \\
\hline 3.76 & 1.62 & -64.17 & -62.54 \\
\hline 3.86 & 0.44 & -60.82 & -60.38 \\
\hline 3.97 & -0.30 & -57.45 & -57.75 \\
\hline 4.07 & -0.73 & -54.09 & -54.82 \\
\hline 4.17 & -0.98 & -50.78 & -51.76 \\
\hline 4.27 & -1.13 & -47.53 & -48.66 \\
\hline 4.37 & -1.20 & -44.39 & -45.59 \\
\hline 4.48 & -1.21 & -41.38 & -42.58 \\
\hline 4.58 & -1.18 & -38.51 & -39.68 \\
\hline 4.68 & -1.12 & -35.80 & -36.92 \\
\hline 4.78 & -1.05 & -33.26 & -34.31 \\
\hline 4.88 & -0.85 & -30.89 & -31.74 \\
\hline
\end{tabular}


Table S8. Adsorption energies from the DFT calculation with the PBE functional, from the vdW correction, and from their addition, denoted as total adsorption $E_{\text {adsorp }}$ for graphene adsorbed on the $\mathrm{Ag}(1,1,1)$ surface at different distances between graphene and metal surface, at optimized cutoff parameter $b=3.30$ Bohr. Units: Angstrom for distance and meV/carbon atom for energy.

\begin{tabular}{lrrr}
\hline$d$ & \multicolumn{1}{l}{$E_{P B E}$} & \multicolumn{1}{c}{$E_{v d W}$} & $E_{\text {adsorp }}$ \\
\hline 2.37 & 213.77 & -140.76 & 73.01 \\
2.47 & 168.20 & -131.83 & 36.37 \\
2.57 & 132.25 & -123.83 & 8.42 \\
2.67 & 103.44 & -116.76 & -13.33 \\
2.77 & 80.10 & -110.55 & -30.45 \\
2.87 & 61.13 & -105.09 & -43.95 \\
2.97 & 45.79 & -100.24 & -54.45 \\
3.07 & 33.52 & -95.88 & -62.36 \\
3.17 & 23.92 & -91.92 & -68.00 \\
3.27 & 16.60 & -88.24 & -71.64 \\
3.37 & 11.15 & -84.76 & -73.61 \\
3.47 & 7.15 & -81.40 & -74.25 \\
3.57 & 4.28 & -78.11 & -73.83 \\
3.67 & 2.24 & -74.82 & -72.58 \\
3.77 & 0.83 & -71.52 & -70.70 \\
3.87 & -0.15 & -68.19 & -68.34 \\
3.97 & -0.80 & -64.82 & -65.62 \\
4.07 & -1.24 & -61.42 & -62.66 \\
4.17 & -1.50 & -58.02 & -59.52 \\
4.27 & -1.63 & -54.64 & -56.27 \\
4.37 & -1.68 & -51.31 & -52.99 \\
4.47 & -1.65 & -48.07 & -49.72 \\
4.57 & -1.57 & -44.94 & -46.52 \\
4.67 & -1.48 & -41.95 & -43.43 \\
4.77 & -1.39 & -39.10 & -40.49 \\
\hline & & &
\end{tabular}


Table S9. Adsorption energies from the DFT calculation with the PBE functional, from the vdW correction, and from their addition, denoted as total adsorption $E_{\text {adsorp }}$ for graphene adsorbed on the $\mathrm{Au}(1,1,1)$ surface at different distances between graphene and metal surface, at optimized cutoff parameter $b=3.30$ Bohr. Units: Angstrom for distance and meV/carbon atom for energy.

\begin{tabular}{lrrr}
\hline$d$ & \multicolumn{1}{l}{$E_{P B E}$} & \multicolumn{1}{c}{$E_{\text {vdW }}$} & $E_{\text {adsorp }}$ \\
\hline 2.12 & 567.04 & -185.75 & 381.29 \\
2.22 & 451.00 & -174.54 & 276.46 \\
2.32 & 358.58 & -163.40 & 195.17 \\
2.42 & 283.62 & -152.93 & 130.68 \\
2.52 & 222.36 & -143.41 & 78.95 \\
2.62 & 172.55 & -134.93 & 37.63 \\
2.72 & 132.58 & -127.44 & 5.13 \\
2.82 & 100.08 & -120.85 & -20.77 \\
2.92 & 73.87 & -115.03 & -41.16 \\
3.02 & 53.53 & -109.83 & -56.30 \\
3.12 & 38.17 & -105.14 & -66.97 \\
3.22 & 26.72 & -100.82 & -74.10 \\
3.32 & 18.29 & -96.78 & -78.49 \\
3.42 & 12.16 & -92.92 & -80.76 \\
3.52 & 7.77 & -89.16 & -81.39 \\
3.62 & 4.66 & -85.45 & -80.79 \\
3.72 & 2.50 & -81.74 & -79.24 \\
3.82 & 1.02 & -77.99 & -76.97 \\
3.92 & 0.03 & -74.21 & -74.18 \\
4.02 & -0.60 & -70.39 & -70.99 \\
4.12 & -0.99 & -66.55 & -67.54 \\
4.22 & -1.21 & -62.73 & -63.94 \\
4.32 & -1.33 & -58.96 & -60.29 \\
4.42 & -1.37 & -55.27 & -56.64 \\
4.52 & -1.37 & -51.69 & -53.07 \\
4.62 & -1.35 & -48.26 & -49.60 \\
\hline & & &
\end{tabular}


Table S10. Physisorption energies (in meV/carbon atom) of graphene on the $(1,1,1)$ surfaces of $\mathrm{Al}, \mathrm{Ni}, \mathrm{Pd}$, $\mathrm{Pt}, \mathrm{Cu}, \mathrm{Ag}, \mathrm{Au}$ and the $(0,0,0,1)$ surface of Co calculated by the method in Refs. [3] and [4], i.e., using the RPA value for $d$. The number of valence electrons of a carbon atom in graphene is chosen to be 4 . The fitted optimal cutoff is $b=3.41$ Bohrs.

\begin{tabular}{ccccccccccc}
\hline \hline & $d$ & $d-c / 2$ & RPA & PBE+vdW & LDA & PBE & vdW-DF & vdW-DF2 & vdW-DF2-C09 & B86bPBE-XDM \\
\hline $\mathrm{Al}$ & 3.51 & 2.34 & 52 & 66 & 29 & 2 & 35 & & & 34 \\
$\mathrm{Ni}$ & 3.26 & 2.24 & 70 & 66 & 50 & 2 & 45 & 44 & 75 & 81 \\
$\mathrm{Co}$ & 3.25 & 2.23 & 66 & 67 & & 2 & 38 & & & \\
$\mathrm{Pd}$ & 3.34 & 2.22 & 90 & 84 & 43 & 4 & 49 & 52 & 72 & 66 \\
$\mathrm{Pt}$ & 3.42 & 2.29 & 84 & 83 & 36 & 5 & 52 & 54 & 68 & 71 \\
$\mathrm{Cu}$ & 3.09 & 2.05 & 68 & 75 & 40 & 2 & 44 & 46 & 62 & 73 \\
$\mathrm{Ag}$ & 3.31 & 2.13 & 78 & 76 & 30 & 2 & 42 & 42 & 53 & 72 \\
$\mathrm{Au}$ & 3.22 & 2.04 & 95 & 86 & 34 & 2 & 47 & 49 & 59 & 66 \\
$\mathrm{ME}$ & & & & 0 & -39 & -74 & -33 & -30 & -16 & -9 \\
$\mathrm{MAE}$ & & & & 5 & 39 & 74 & 33 & 30 & 19 & 17 \\
\hline
\end{tabular}


Table S11. The ratio of vdW adsorption energy from the $C_{5}$ term (denoted vdW5) to the total $\mathrm{vdW}$ adsorption energy (denoted $\mathrm{vdW} 3+\mathrm{vdW} 5$ ), and the damping function $f_{d}$ at equilibrium. $d$ $(\mathrm{PBE}+\mathrm{vdW})$ is the equilibrium distance between graphene and metal surface at the optimal $b=3.30$ Bohrs, determined from the adsorption energy curve of the PBE calculation plus the vdW model as a function of distance, as explained in text. $c / 2$ determines the background edge of the metal as explained in text. All entries are in atomic units.

\begin{tabular}{|c|c|c|c|c|c|c|c|}
\hline Metals & $C_{3}$ & $C_{5}$ & $Z_{0}$ & $d_{(\mathrm{PBE}+\mathrm{vdW})}-c / 2$ & vdW5/(vdW3+vdW5) & $x=\left(Z-Z_{0}\right) / b$ & $f_{d}$ \\
\hline $\mathrm{Al}$ & 0.326 & 2.369 & 0.876 & 4.640 & 0.34 & 1.14 & 0.28 \\
\hline $\mathrm{Ni}$ & 0.329 & 2.480 & 0.421 & 4.306 & 0.33 & 1.18 & 0.32 \\
\hline Co & 0.332 & 2.507 & 0.412 & 4.875 & 0.27 & 1.35 & 0.46 \\
\hline $\mathrm{Pd}$ & 0.424 & 3.181 & 0.269 & 3.915 & 0.36 & 1.10 & 0.26 \\
\hline $\mathrm{Pt}$ & 0.428 & 3.205 & 0.268 & 4.451 & 0.30 & 1.27 & 0.39 \\
\hline $\mathrm{Cu}$ & 0.355 & 2.670 & 0.369 & 4.717 & 0.28 & 1.32 & 0.43 \\
\hline $\mathrm{Ag}$ & 0.382 & 2.810 & 0.369 & 4.313 & 0.32 & 1.20 & 0.33 \\
\hline $\mathrm{Au}$ & 0.419 & 3.126 & 0.294 & 4.318 & 0.32 & 1.22 & 0.35 \\
\hline
\end{tabular}


Table S12. Adsorption energies from the DFT-PBE, the vdW correction, and their addition, denoted as total adsorption $E_{\text {adsorp }}$ for graphene adsorbed on the $\mathrm{Al}(1,1,1)$ surface at different distances between graphene and metal surface, at optimized cutoff parameter $b=3.28$ Bohr. Units: Angstrom for distance and $\mathrm{meV} /$ carbon atom for energy. The data points of $E_{P B E}$ are from Ref. (5).

\begin{tabular}{rlrl}
\hline \hline$d$ & $E_{P B E}$ & $E_{v d W}$ & $E_{\text {adsorp }}$ \\
\hline 3.05 & 42.13 & -92.88 & -50.75 \\
3.11 & 35.11 & -90.11 & -55.00 \\
3.17 & 28.96 & -87.53 & -58.57 \\
3.23 & 23.63 & -85.11 & -61.49 \\
3.29 & 19.06 & -82.85 & -63.79 \\
3.35 & 15.18 & -80.71 & -65.53 \\
3.42 & 11.94 & -78.67 & -66.74 \\
3.48 & 9.24 & -76.72 & -67.48 \\
3.54 & 7.04 & -74.83 & -67.80 \\
3.60 & 5.25 & -73.00 & -67.75 \\
3.66 & 3.82 & -71.20 & -67.38 \\
3.72 & 2.68 & -69.42 & -66.74 \\
3.78 & 1.79 & -67.65 & -65.87 \\
3.85 & 1.09 & -65.89 & -64.81 \\
3.91 & 0.54 & -64.13 & -63.59 \\
3.97 & 0.10 & -62.35 & -62.25 \\
4.03 & -0.25 & -60.57 & -60.82 \\
4.09 & -0.53 & -58.77 & -59.30 \\
4.15 & -0.77 & -56.96 & -57.73 \\
4.22 & -0.98 & -55.14 & -56.12 \\
4.28 & -1.15 & -53.31 & -54.47 \\
4.34 & -1.31 & -51.49 & -52.79 \\
4.40 & -1.43 & -49.66 & -51.10 \\
4.46 & -1.53 & -47.85 & -49.38 \\
4.52 & -1.60 & -46.05 & -47.65 \\
4.58 & -1.64 & -44.28 & -45.91 \\
4.65 & -1.64 & -42.53 & -44.17 \\
4.71 & -1.60 & -40.82 & -42.42 \\
4.77 & -1.54 & -39.15 & -40.69 \\
4.83 & -1.46 & -37.52 & -38.98 \\
4.89 & -1.37 & -35.94 & -37.32 \\
\hline & & &
\end{tabular}


Table S13. Adsorption energies from the DFT-PBE, the vdW correction, and their addition, denoted as total adsorption $E_{\text {adsorp }}$ for graphene adsorbed on the Ni $(1,1,1)$ surface at different distances between graphene and metal surface, at optimized cutoff parameter $b=3.28$ Bohr. Units: Angstrom for distance and $\mathrm{meV} /$ carbon atom for energy. The data points of $E_{P B E}$ are from Ref. (5).

\begin{tabular}{rrrr}
\hline \hline$d$ & $E_{P B E}$ & $E_{v d W}$ & $E_{\text {adsorp }}$ \\
\hline 2.85 & 30.01 & -90.45 & -60.44 \\
2.92 & 26.20 & -87.74 & -61.55 \\
2.99 & 22.25 & -85.20 & -62.95 \\
3.05 & 18.42 & -82.78 & -64.36 \\
3.12 & 14.88 & -80.46 & -65.58 \\
3.19 & 11.72 & -78.22 & -66.50 \\
3.26 & 8.97 & -76.03 & -67.06 \\
3.32 & 6.65 & -73.87 & -67.22 \\
3.39 & 4.73 & -71.74 & -67.01 \\
3.46 & 3.16 & -69.61 & -66.45 \\
3.53 & 1.91 & -67.48 & -65.58 \\
3.60 & 0.91 & -65.35 & -64.43 \\
3.66 & 0.13 & -63.19 & -63.06 \\
3.73 & -0.49 & -61.03 & -61.51 \\
3.80 & -0.97 & -58.85 & -59.82 \\
3.87 & -1.35 & -56.67 & -58.01 \\
3.93 & -1.64 & -54.48 & -56.12 \\
4.00 & -1.86 & -52.31 & -54.17 \\
4.07 & -2.02 & -50.15 & -52.17 \\
4.14 & -2.13 & -48.02 & -50.15 \\
4.20 & -2.19 & -45.92 & -48.11 \\
4.27 & -2.20 & -43.87 & -46.07 \\
4.34 & -2.18 & -41.87 & -44.05 \\
4.41 & -2.13 & -39.93 & -42.05 \\
4.48 & -2.06 & -38.05 & -40.11 \\
4.54 & -2.00 & -36.24 & -38.24 \\
4.61 & -1.95 & -34.50 & -36.45 \\
4.68 & -1.92 & -32.84 & -34.76 \\
4.75 & -1.93 & -31.25 & -33.18 \\
4.81 & -1.96 & -29.73 & -31.69 \\
4.88 & -1.99 & -28.29 & -30.27 \\
\hline & & &
\end{tabular}


Table S14. Adsorption energies from the DFT-PBE, the vdW correction, and their addition, denoted as total adsorption $E_{\text {adsorp }}$ for graphene adsorbed on the Co $(0,0,0,1)$ surface at different distances between graphene and metal surface, at optimized cutoff parameter $b=3.28$ Bohr. Units: Angstrom for distance and $\mathrm{meV} /$ carbon atom for energy. The data points of $E_{P B E}$ are from Ref. (5).

\begin{tabular}{rlrl}
\hline \hline$d$ & $E_{P B E}$ & $E_{v d W}$ & $E_{\text {adsorp }}$ \\
\hline 3.10 & 16.89 & -80.91 & -64.02 \\
3.18 & 13.83 & -78.25 & -64.42 \\
3.26 & 10.84 & -75.66 & -64.82 \\
3.34 & 8.09 & -73.11 & -65.02 \\
3.42 & 5.66 & -70.56 & -64.91 \\
3.50 & 3.62 & -68.02 & -64.40 \\
3.58 & 1.98 & -65.46 & -63.49 \\
3.66 & 0.72 & -62.89 & -62.17 \\
3.74 & -0.20 & -60.29 & -60.49 \\
3.82 & -0.84 & -57.68 & -58.52 \\
3.90 & -1.26 & -55.08 & -56.33 \\
3.98 & -1.52 & -52.48 & -54.00 \\
4.06 & -1.69 & -49.91 & -51.60 \\
4.14 & -1.82 & -47.38 & -49.20 \\
4.22 & -1.93 & -44.91 & -46.84 \\
4.30 & -2.03 & -42.51 & -44.54 \\
4.38 & -2.13 & -40.19 & -42.32 \\
4.46 & -2.19 & -37.96 & -40.15 \\
4.54 & -2.19 & -35.83 & -38.02 \\
4.62 & -2.10 & -33.80 & -35.90 \\
4.70 & -1.91 & -31.88 & -33.79 \\
4.78 & -1.66 & -30.06 & -31.72 \\
4.86 & -1.42 & -28.34 & -29.77 \\
\hline & & &
\end{tabular}


Table S15. Adsorption energies from the DFT-PBE, the vdW correction, and their addition, denoted as total adsorption $E_{\text {adsorp }}$ for graphene adsorbed on the $\mathrm{Pd}(1,1,1)$ surface at different distances between graphene and metal surface, at optimized cutoff parameter $b=3.28$ Bohr. Units: Angstrom for distance and $\mathrm{meV} /$ carbon atom for energy. The data points of $E_{P B E}$ are from Ref. (5).

\begin{tabular}{rccc}
\hline \hline$d$ & $E_{P B E}$ & $E_{v d W}$ & $E_{\text {adsorp }}$ \\
\hline 2.85 & 42.01 & -119.09 & -77.08 \\
2.92 & 34.63 & -115.43 & -80.80 \\
2.99 & 28.12 & -112.01 & -83.89 \\
3.05 & 22.44 & -108.78 & -86.33 \\
3.12 & 17.55 & -105.71 & -88.15 \\
3.19 & 13.39 & -102.76 & -89.37 \\
3.26 & 9.90 & -99.92 & -90.02 \\
3.32 & 6.99 & -97.13 & -90.15 \\
3.39 & 4.59 & -94.40 & -89.80 \\
3.46 & 2.65 & -91.69 & -89.04 \\
3.53 & 1.08 & -88.98 & -87.90 \\
3.60 & -0.16 & -86.27 & -86.44 \\
3.66 & -1.14 & -83.55 & -84.69 \\
3.73 & -1.90 & -80.81 & -82.71 \\
3.80 & -2.48 & -78.05 & -80.53 \\
3.87 & -2.91 & -75.27 & -78.18 \\
3.93 & -3.22 & -72.49 & -75.71 \\
4.00 & -3.44 & -69.71 & -73.14 \\
4.07 & -3.57 & -66.93 & -70.51 \\
4.14 & -3.64 & -64.18 & -67.83 \\
4.20 & -3.67 & -61.47 & -65.13 \\
4.27 & -3.65 & -58.79 & -62.44 \\
4.34 & -3.60 & -56.17 & -59.77 \\
4.41 & -3.53 & -53.62 & -57.15 \\
4.48 & -3.44 & -51.14 & -54.59 \\
4.54 & -3.36 & -48.75 & -52.10 \\
4.61 & -3.27 & -46.44 & -49.71 \\
4.68 & -3.18 & -44.22 & -47.40 \\
4.75 & -3.09 & -42.09 & -45.18 \\
4.81 & -2.99 & -40.06 & -43.05 \\
4.88 & -2.85 & -38.12 & -40.97 \\
\hline & & &
\end{tabular}


Table S16. Adsorption energies from the DFT-PBE, the vdW correction, and their addition, denoted as total adsorption $E_{\text {adsorp }}$ for graphene adsorbed on the $\mathrm{Pt}(1,1,1)$ surface at different distances between graphene and metal surface, at optimized cutoff parameter $b=3.28$ Bohr. Units: Angstrom for distance and $\mathrm{meV} /$ carbon atom for energy. The data points of $E_{P B E}$ are from Ref. (5).

\begin{tabular}{rlll}
\hline \hline$d$ & $E_{P B E}$ & $E_{v d W}$ & $E_{\text {adsorp }}$ \\
\hline 3.12 & 34.74 & -107.02 & -72.28 \\
3.18 & 28.33 & -104.41 & -76.08 \\
3.24 & 22.64 & -101.88 & -79.24 \\
3.30 & 17.70 & -99.41 & -81.71 \\
3.36 & 13.47 & -96.98 & -83.51 \\
3.42 & 9.91 & -94.59 & -84.68 \\
3.48 & 6.95 & -92.21 & -85.26 \\
3.54 & 4.51 & -89.83 & -85.33 \\
3.60 & 2.51 & -87.45 & -84.94 \\
3.66 & 0.89 & -85.06 & -84.17 \\
3.72 & -0.42 & -82.66 & -83.08 \\
3.77 & -1.48 & -80.25 & -81.73 \\
3.83 & -2.34 & -77.82 & -80.16 \\
3.89 & -3.05 & -75.38 & -78.43 \\
3.95 & -3.64 & -72.93 & -76.57 \\
4.01 & -4.11 & -70.49 & -74.60 \\
4.07 & -4.50 & -68.05 & -72.55 \\
4.13 & -4.81 & -65.63 & -70.43 \\
4.19 & -5.03 & -63.23 & -68.26 \\
4.25 & -5.18 & -60.85 & -66.04 \\
4.31 & -5.26 & -58.52 & -63.78 \\
4.36 & -5.26 & -56.23 & -61.49 \\
4.42 & -5.19 & -53.99 & -59.19 \\
4.48 & -5.07 & -51.81 & -56.88 \\
4.54 & -4.90 & -49.69 & -54.59 \\
4.60 & -4.70 & -47.64 & -52.34 \\
4.66 & -4.49 & -45.65 & -50.14 \\
4.72 & -4.30 & -43.74 & -48.03 \\
4.78 & -4.14 & -41.89 & -46.03 \\
4.84 & -4.03 & -40.12 & -44.15 \\
4.90 & -3.97 & -38.42 & -42.39 \\
\hline & & &
\end{tabular}


Table S17. Adsorption energies from the DFT-PBE, the vdW correction, and their addition, denoted as total adsorption $E_{\text {adsorp }}$ for graphene adsorbed on the $\mathrm{Cu}(1,1,1)$ surface at different distances between graphene and metal surface, at optimized cutoff parameter $b=3.28$ Bohr. Units: Angstrom for distance and meV/carbon atom for energy. The data points of $E_{P B E}$ are from Ref. (5).

\begin{tabular}{rlll}
\hline \hline$d$ & $E_{P B E}$ & $E_{v d W}$ & $E_{\text {adsorp }}$ \\
\hline 2.75 & 44.06 & -103.20 & -59.14 \\
2.82 & 38.65 & -99.75 & -61.10 \\
2.89 & 33.20 & -96.56 & -63.35 \\
2.97 & 27.98 & -93.57 & -65.60 \\
3.04 & 23.14 & -90.76 & -67.62 \\
3.11 & 18.79 & -88.09 & -69.30 \\
3.18 & 14.97 & -85.53 & -70.56 \\
3.25 & 11.68 & -83.04 & -71.36 \\
3.32 & 8.90 & -80.61 & -71.71 \\
3.39 & 6.58 & -78.21 & -71.63 \\
3.46 & 4.68 & -75.82 & -71.15 \\
3.53 & 3.13 & -73.44 & -70.31 \\
3.60 & 1.88 & -71.05 & -69.17 \\
3.67 & 0.87 & -68.64 & -67.76 \\
3.74 & 0.08 & -66.21 & -66.14 \\
3.81 & -0.56 & -63.77 & -64.33 \\
3.89 & -1.05 & -61.32 & -62.37 \\
3.96 & -1.43 & -58.87 & -60.30 \\
4.03 & -1.71 & -56.43 & -58.14 \\
4.10 & -1.91 & -54.01 & -55.92 \\
4.17 & -2.04 & -51.62 & -53.66 \\
4.24 & -2.11 & -49.26 & -51.38 \\
4.31 & -2.13 & -46.97 & -49.09 \\
4.38 & -2.11 & -44.73 & -46.84 \\
4.45 & -2.06 & -42.56 & -44.62 \\
4.52 & -2.01 & -40.47 & -42.47 \\
4.59 & -1.96 & -38.46 & -40.41 \\
4.66 & -1.93 & -36.53 & -38.45 \\
4.73 & -1.92 & -34.69 & -36.61 \\
4.81 & -1.93 & -32.94 & -34.86 \\
4.88 & -1.93 & -31.27 & -33.20 \\
4.95 & -1.88 & -29.69 & -31.56 \\
\hline & & &
\end{tabular}


Table S18. Adsorption energies from the DFT-PBE, the vdW correction, and their addition, denoted as total adsorption $E_{\text {adsorp }}$ for graphene adsorbed on the $\mathrm{Ag}(1,1,1)$ surface at different distances between graphene and metal surface, at optimized cutoff parameter $b=3.28$ Bohr. Units: Angstrom for distance and $\mathrm{meV} /$ carbon atom for energy. The data points of $E_{P B E}$ are from Ref. (5).

\begin{tabular}{rlll}
\hline$d$ & $E_{P B E}$ & \multicolumn{1}{c}{$E_{v d W}$} & $E_{\text {adsorp }}$ \\
\hline 2.37 & 213.77 & -145.10 & 68.67 \\
2.47 & 168.20 & -135.89 & 32.31 \\
2.57 & 132.25 & -127.64 & 4.61 \\
2.67 & 103.44 & -120.35 & -16.91 \\
2.77 & 80.10 & -113.94 & -33.83 \\
2.87 & 61.13 & -108.28 & -47.15 \\
2.97 & 45.79 & -103.26 & -57.48 \\
3.07 & 33.52 & -98.75 & -65.23 \\
3.17 & 23.92 & -94.63 & -70.71 \\
3.27 & 16.60 & -90.79 & -74.19 \\
3.37 & 11.15 & -87.16 & -76.01 \\
3.47 & 7.15 & -83.64 & -76.48 \\
3.57 & 4.28 & -80.17 & -75.89 \\
3.67 & 2.24 & -76.72 & -74.47 \\
3.77 & 0.83 & -73.24 & -72.41 \\
3.87 & -0.15 & -69.73 & -69.87 \\
3.97 & -0.80 & -66.18 & -66.98 \\
4.07 & -1.24 & -62.61 & -63.84 \\
4.17 & -1.50 & -59.04 & -60.55 \\
4.27 & -1.63 & -55.52 & -57.15 \\
4.37 & -1.68 & -52.05 & -53.73 \\
4.47 & -1.65 & -48.69 & -50.34 \\
4.57 & -1.57 & -45.46 & -47.03 \\
4.67 & -1.48 & -42.37 & -43.86 \\
4.77 & -1.39 & -39.45 & -40.84 \\
\hline & & &
\end{tabular}


Table S19. Adsorption energies from the DFT-PBE, the vdW correction, and their addition, denoted as total adsorption $E_{\text {adsorp }}$ for graphene adsorbed on the $\mathrm{Au}(1,1,1)$ surface at different distances between graphene and metal surface, at optimized cutoff parameter $b=3.28$ Bohr. Units: Angstrom for distance and $\mathrm{meV} /$ carbon atom for energy. The data points of $E_{P B E}$ are from Ref. (5).

\begin{tabular}{rlrl}
\hline$d$ & $E_{P B E}$ & $E_{v d W}$ & $E_{\text {adsorp }}$ \\
\hline 2.12 & 567.04 & -191.48 & 375.56 \\
2.22 & 451.00 & -179.92 & 271.08 \\
2.32 & 358.58 & -168.45 & 190.13 \\
2.42 & 283.62 & -157.65 & 125.97 \\
2.52 & 222.36 & -147.83 & 74.54 \\
2.62 & 172.55 & -139.07 & 33.48 \\
2.72 & 132.58 & -131.35 & 1.23 \\
2.82 & 100.08 & -124.54 & -24.46 \\
2.92 & 73.87 & -118.52 & -44.65 \\
3.02 & 53.53 & -113.14 & -59.61 \\
3.12 & 38.17 & -108.26 & -70.09 \\
3.22 & 26.72 & -103.77 & -77.05 \\
3.32 & 18.29 & -99.55 & -81.26 \\
3.42 & 12.16 & -95.51 & -83.35 \\
3.52 & 7.77 & -91.57 & -83.80 \\
3.62 & 4.66 & -87.66 & -83.00 \\
3.72 & 2.50 & -83.75 & -81.25 \\
3.82 & 1.02 & -79.80 & -78.79 \\
3.92 & 0.03 & -75.82 & -75.79 \\
4.02 & -0.60 & -71.80 & -72.41 \\
4.12 & -0.99 & -67.78 & -68.77 \\
4.22 & -1.21 & -63.79 & -65.00 \\
4.32 & -1.33 & -59.85 & -61.18 \\
4.42 & -1.37 & -56.02 & -57.39 \\
4.52 & -1.37 & -52.32 & -53.69 \\
4.62 & -1.35 & -48.77 & -50.12 \\
\hline & & &
\end{tabular}


Table S20. Physisorption energies (in meV/carbon atom) and equilibrium distances (in Angstrom) of graphene on the $(1,1,1)$ surfaces of $\mathrm{Al}, \mathrm{Ni}, \mathrm{Pd}, \mathrm{Pt}, \mathrm{Cu}, \mathrm{Ag}$, $\mathrm{Au}$ and the $(0,0,0,1)$ surface of $\mathrm{Co}$ calculated by using the PBE results from the Ref. (5). The number of valence electrons of a carbon atom in graphene is 4 . The fitted optimal cutoff is $b=3.28$ Bohrs.

\begin{tabular}{ccccc}
\hline \hline Metals & $E_{R P A}$ & $E_{P B E+v d W}$ & $d_{R P A}$ & $d_{P B E+v d W}$ \\
\hline $\mathrm{Al}$ & 52 & 67.8 & 3.51 & 3.54 \\
$\mathrm{Ni}$ & 70 & 67.2 & 3.26 & 3.32 \\
$\mathrm{Co}$ & 66 & 65.0 & 3.25 & 3.34 \\
$\mathrm{Pd}$ & 90 & 90.2 & 3.34 & 3.32 \\
$\mathrm{Pt}$ & 84 & 85.3 & 3.42 & 3.54 \\
$\mathrm{Cu}$ & 68 & 71.7 & 3.09 & 3.32 \\
$\mathrm{Ag}$ & 78 & 76.5 & 3.31 & 3.47 \\
$\mathrm{Au}$ & 95 & 83.8 & 3.22 & 3.52 \\
\hline $\mathrm{ME}$ & & 0.6 & & 0.12 \\
$\mathrm{MAE}$ & & 4.7 & & 0.13 \\
\hline
\end{tabular}




\section{References}

[1] Persson, B. N. J.; Zaremba, E. Reference-Plane Position for the Atom-Surface van der Waals Interaction. Phys. Rev. B 1984, 30, 5669.

[2] Tao, J.; Rappe, A.M. Physical Adsorption: Theory of van der Waals Interactions between Particles and Clean Surfaces. Phys. Rev. Lett. 2014, 112, 106101.

[3] Tao, J.; Tang, H.; Patra, A.; Bhattarai, P.; Perdew, J. P. Modeling the Physisorption of Graphene on Metals. Phys. Rev. B 2018, 97, 165403.

[4] Tao, J.; Tang, H.; Patra, A.; Bhattarai, P.; Perdew, J. P. Erratum: Modeling the Physisorption of Graphene on Metals [Phys. Rev. B 97, 165403 (2018)]. Phys. Rev. B 2019, 99, 169901(E).

[5] Olsen,T.; Thygesen, K. S. Random Phase Approximation Applied to Solids, Molecules, and Graphene-Metal Interfaces: From van der Waals to Covalent Bonding. Phys. Rev. B 2013, $87,075111$. 